\title{
DETECTING BILATERAL FUNCTIONAL CONNECTIVITY IN THE PREFRONTAL CORTEX DURING A STROOP TASK BY NEAR-INFRARED SPECTROSCOPY
}

\author{
LEI ZHANG ${ }^{*, \dagger}, \S$, JINYAN SUN ${ }^{*, \dagger, \S}$, BAILEI SUN ${ }^{*, \dagger}$, \\ CHENYANG GAO ${ }^{*, \dagger}$ and HUI GONG ${ }^{*, \dagger, *}$ \\ *Britton Chance Center for Biomedical Photonics \\ Wuhan National Laboratory for Optoelectronics \\ Huazhong University of Science and Technology \\ Wuhan 430074, P. R. China \\ ${ }^{\dagger}$ MoE Key Laboratory for Biomedical Photonics \\ Department of Biomedical Engineering \\ Huazhong University of Science and Technology \\ Wuhan 430074, P. R. China \\ \$huigong@mail.hust.edu.cn
}

Received 30 May 2013

Accepted 18 July 2013

Published 22 August 2013

\begin{abstract}
Near-infrared spectroscopy (NIRS) is generally accepted as a functional brain imaging technology for brain activation study. With multichannel highly sensitive NIRS instruments, it has become possible to assess functional connectivity of different brain regions by NIRS. However, the feasibility needs to be validated in complex cognitive activities. In this study, we recorded the hemodynamic activity of the bilateral prefrontal cortex (PFC) during a color-word matching Stroop task. Wavelet transform coherence (WTC) analysis was applied to assess the functional connectivity of all homologous channel pairs within the left/right PFC. Both the behavioral and brain activation results showed significant Stroop effects. The results of WTC analysis revealed that, bilateral functional connectivity was significantly stronger during both the incongruent stimuli and neutral stimuli compared to that of the rest period. It also showed significant Stroop effect. Our findings demonstrate that, NIRS becomes a valuable tool to elucidate the functional connectivity of brain cortex in complex cognitive activities.
\end{abstract}

Keywords: Near-infrared spectroscopy; bilateral functional connectivity; wavelet transform coherence; complex cognitive activities.

$\S$ These authors contributed equally to this work.

This is an Open Access article published by World Scientific Publishing Company. It is distributed under the terms of the Creative Commons Attribution 3.0 (CC-BY) License. Further distribution of this work is permitted, provided the original work is properly cited. 


\section{Introduction}

Near-infrared spectroscopy (NIRS) is a generally accepted functional brain imaging technology which monitors brain activity noninvasively by measuring hemodynamic responses. ${ }^{1-3}$ Compared to other traditional neuroimaging modalities, such as electroencephalography (EEG)/event related potential (ERP) and functional magnetic resonance imaging (fMRI), NIRS can provide a excellent temporal resolution and a reasonable spatial resolution at the same time, ${ }^{4,5}$ with fewer requirements for the environment and participants. ${ }^{6-8}$ Traditionally, NIRS is used to assess local brain activity and has been applied in various cognitive and clinical studies. ${ }^{9-13}$ With the developments of multichannel highly sensitive NIRS instruments, it has also become possible to measure temporal correlations of local activity at different brain areas, deriving an optical "functional connectivity". ${ }^{14}$

In recent years, there have been some studies that investigated and preliminarily validated the feasibility of NIRS in studying functional connectivity. ${ }^{15-17}$ However, most of these studies are about resting state functional connectivity (RSFC), which reflects interactions of spontaneous neuronal activity among different brain regions at the resting state. ${ }^{16}$ As to cognitive functions, which are very important for dealing with various information of daily life, there are few functional connectivity studies with NIRS. In order to promote application of NIRS-based functional connectivity, it is necessary to conduct more studies to explore the feasibility of NIRS in high-level and complex cognitive tasks.

Cognitive control is one of the important cognitive functions of human beings. It refers to the ability to ignore the distracting information, attend to the goal-relevant information, overcome conflict as well as select the appropriate response. ${ }^{18}$ The Stroop task is widely used to study the cognitive control over conflicts. ${ }^{19,20}$ Existing brain functional imaging researches demonstrate that prefrontal cortex (PFC) plays a predominant role in the Stroop task, through biasing the processing of taskrelated information to resolve conflict. ${ }^{21,22}$

In the present study, we aimed to examine if NIRS can be used to study the functional connectivity in a Stroop task. A Chinese color-word matching Stroop task that primarily activates the bilateral PFC was employed. ${ }^{23-25}$ The hemodynamic signal from PFC and behavioral data were recorded simultaneously. Wavelet transform coherence (WTC) analysis was used to assess the NIRSbased functional connectivity between left and right PFC. The Stroop effects of behavioral performance, brain activation and bilateral functional connectivity were explored to study the feasibility of imaging NIRS-based functional connectivity in high-level and complex cognitive tasks.

\section{Methods}

\subsection{Subjects}

A total of 12 right-handed paid volunteers (3 females) aged 20-27 years [mean, 23.5 years; standard deviation (SD), 2.11 years] participated in the study. All participants had normal color vision and normal (or corrected-to-normal) vision. All volunteers were healthy without any neurological or psychiatric disorders. Written informed consent was obtained from each participant before the experiment, and the study was approved by the Human Subjects Institutional Review Board of Huazhong University of Science and Technology.

\subsection{Procedures}

We used the color-word matching Stroop task with a block design, which was adapted from previous studies. ${ }^{23,24}$ There were two Chinese characters in each stimulus, and subjects were asked to judge if the color of the upper Chinese character was consistent with the meaning of the lower Chinese character [see Fig. 1(a)]. If "Yes", participants pressed a button with their left index finger, and if "No", they pressed another button with their right index finger. There were two kinds of stimulus conditions: neutral and incongruent. In neutral stimuli, the upper Chinese character was a noncolor word (涂, 贯, 华, 球, 奖, meaning "scrawl", “pass through", "China", "ball", "prize") presented in green, yellow, red, blue or purple, and the lower Chinese character was a color word (红, 黄, 蓝, 绿, 紫, meaning "red", "yellow", "blue", "green" and "purple") presented in white. In incongruent stimuli, the upper Chinese character was a color word printed in a disparate color [see Fig. 1(a)]. In order to prevent participants from ignoring the upper Chinese character, we asked them to attend to the upper Chinese character first before making the judgment. For each stimulus condition, the 


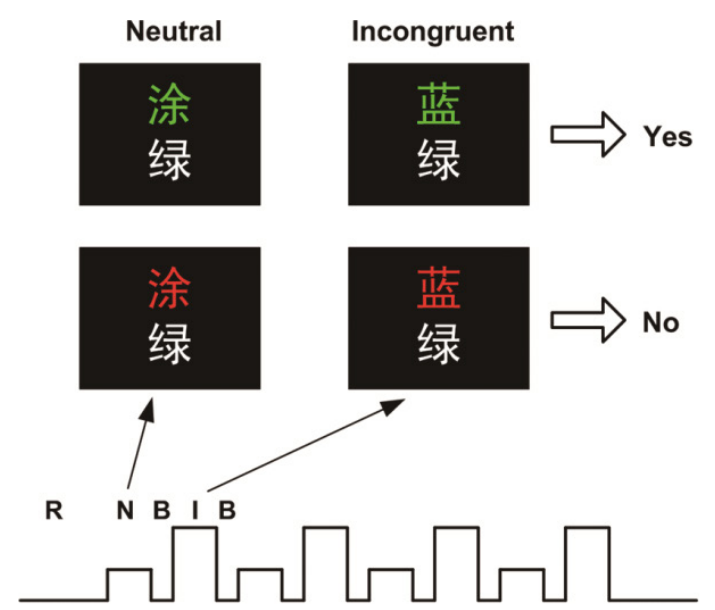

(a)

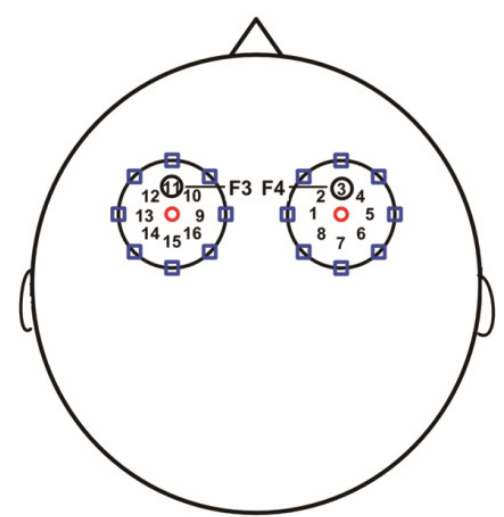

(b)

Fig. 1. (a) Examples of two stimulus conditions (top) and experimental design (bottom). 蓝, 绿 correspond to "blue", "green" and 涂 means "print". The bottom subgraph shows the blocks sequence in a run. R, 60s rest period between runs; B, 15s baseline between block; N, 30 s neutral block; I, 30 s incongruent block. (b) Position of the two NIRS probes on the head. The blue rectangle indicates the detector, and the red circle indicates the source. The number (1-16) at the midpoint of source-detector pair denotes the NIRS detector channel. Channel 3 was over F4 EEG electrode position and Channel 11 was over F3 EEG electrode position.

numbers of "Yes" trials and "No" trials were equal, and the two kinds of trials were semi-randomly mixed in order to avoid the consecutive appearance of more than three trials in the same category.

There were four runs in the experiment. Each run was composed of eight blocks (four neutral task blocks alternated with four incongruent task blocks) [see Fig. 1(a)]. Each block contained 20 trials. In each trial, a stimulus was shown for $1200 \mathrm{~ms}$, followed by a blank screen of $300 \mathrm{~ms}$. The rest period lasted $60 \mathrm{~s}$ between runs, and $15 \mathrm{~s}$ between blocks. There was also a $60 \mathrm{~s}$ rest period before the first run and after the fourth run. During the rest period, a white cross appeared in the center of the screen. One second before a block, there was a sound to cue subjects. Every subject had a practice session before the formal experiment.

\subsection{NIRS recording}

We used a homemade continuous-wave NIRS system to record the concentration changes of oxy-hemoglobin $\left(\Delta\left[\mathrm{HbO}_{2}\right]\right)$ and deoxy-hemoglobin $(\Delta[\mathrm{Hb}]) .{ }^{26}$ There were two probes, each of which held one source $(785$ and $850 \mathrm{~nm})$ and eight detectors to provide eight source-detector pairs. The detector channels were defined as midpoints of the source-detector pairs, so each probe contained eight detector channels. Those two probes were used to cover bilateral PFC. The channel (Ch.) 3 of the right probe was at the location of $\mathrm{F} 4$ electrode, and the left probe was placed symmetrically [see Fig. 1(b)]. The distance of each source-detector pair was $3 \mathrm{~cm}$, and the temporal resolution we adopted was $70 \mathrm{~Hz}$.

\subsection{Behavioral data analysis}

The mean number of trials with no responses averaged across subjects were less than 1 for both stimulus conditions, and these missed trials were not used for the behavioral data analysis. For response time analysis, only trials with response time lying within three standard deviations of the mean value were used. Two paired $t$-tests were performed for response time and false rate, across the two stimulus conditions.

\subsection{NIRS data analysis}

Since $\mathrm{HbO}_{2}$ has a better signal-to-noise ratio (SNR) than $\mathrm{Hb},{ }^{27,28}$ we employed $\mathrm{HbO}_{2}$ to estimate the changes of cerebral blood oxygenation.

\subsubsection{Brain activation analysis}

The raw NIRS data were low-pass filtered at $3 \mathrm{~Hz}$ to remove instrument noise, and downsampled to 
$10 \mathrm{~Hz}$. After converting to change in optical density, the data were band-pass filtered with a frequency range from 0.015 to $0.5 \mathrm{~Hz}$ to eliminate arterial pulse oscillations and slow drifts. Then we used the differential pathlength factor (DPF) method to convert the optical intensity data into $\mathrm{HbO}_{2}$ signals, and the DPF values used here were 5.2 at $850 \mathrm{~nm}$ and 6.0 at $785 \mathrm{~nm} .{ }^{29}$ We used the wavelet minimum description length detrending algorithm to suppress unknown global trends. Finally, the $\mathrm{HbO}_{2}$ signals were block averaged.

The mean value of $\mathrm{HbO}_{2}$ signal of the last $5 \mathrm{~s}$ of rest period before the task was computed for each subject, channel and task condition as baseline. The mean value of $\mathrm{HbO}_{2}$ signal of $0-30 \mathrm{~s}$ after the task's beginning was computed as task response. The difference between the task response and baseline indicated the $\mathrm{HbO}_{2}$ response to each task condition. Paired $t$-test was performed to compare $\mathrm{HbO}_{2}$ response between the two task conditions.

\subsubsection{Functional connectivity analysis}

WTC, also named Wavelet coherence, measures the cross-correlation of two time series as a function of time and frequency. ${ }^{30}$ This method has the ability to uncover the locally phase-locked behavior, which might be difficult to be discovered by traditional time series analysis. ${ }^{31}$ More thorough explanations of WTC were described by Grinsted et al., ${ }^{32}$ and we employed the WTC MATLAB package provided on their website ("http://noc.ac.uk/using-science/ crosswavelet-wavelet-coherence") to evaluate functional connectivity.

Firstly, raw optical data were low-pass filtered at $3 \mathrm{~Hz}$, downsampled to $10 \mathrm{~Hz}$, and converted to optical density. Then the optical intensity data were converted into $\mathrm{HbO}_{2}$ signals with DPF method. The $\mathrm{HbO}_{2}$ signals were block averaged to obtain the $\mathrm{HbO}_{2}$ time series. Subsequent analysis was implemented similarly as described by Cui et al. ${ }^{31}$ WTC was employed to measure relationships between the $\mathrm{HbO}_{2}$ time series within each homologous channel pair. WTC analysis on each pair of the $\mathrm{HbO}_{2}$ time series generated three two-dimensional (2D) coherence maps for three conditions (e.g., Fig. 4). A frequency band associated with tasks was identified, which was between 0.07 and $0.50 \mathrm{~Hz}$ (corresponding to period 14 and $2 \mathrm{~s}$, respectively), and the average coherence value in this band was calculated. Paired $t$-tests were performed for each channel pair between each two conditions (rest period vs neutral task, rest period vs incongruent task and neutral task vs incongruent task). Prior to paired $t$-test, Fisher's z-transform was employed. ${ }^{31}$

\section{Results}

\subsection{Behavioral results}

Figure 2 displays the average response times and average false rates for the two stimulus conditions. The behavioral results suggested longer response time for incongruent condition than neutral condition, and the difference was significant $(p<0.01)$. As to false rate, there was also significant difference $(p<0.01)$ between the two stimulus conditions, and it was higher for incongruent condition. Both response time and false rate showed significant Stroop effects.

\subsection{Brain activation results}

Figure 3 displays the typical time course of $\mathrm{HbO}_{2}$ signal for the two kinds of task conditions. There were significant Stroop effects in Ch. 2, Ch. 3, Ch. 4, Ch. 6, Ch. 9, Ch. 10, Ch. 11, Ch. 12, Ch. 13, Ch. 14, Ch. 15 and Ch. $16(p<0.05)$, with higher $\mathrm{HbO}_{2}$ response for incongruent task than neutral task.

\subsection{Functional connectivity results}

Figure 4 shows the three 2D coherence maps at one typical channel pair of a subject. Coherence increase associated with tasks was found in the frequency

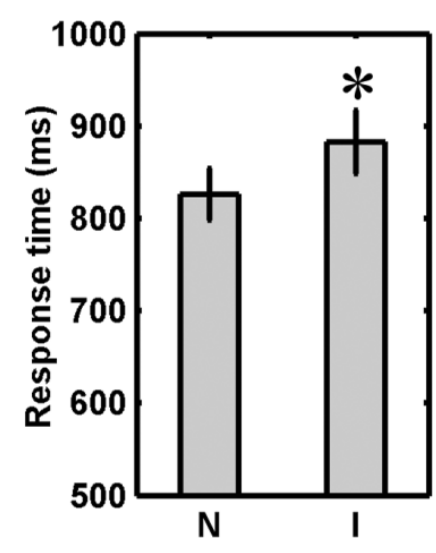

(a)

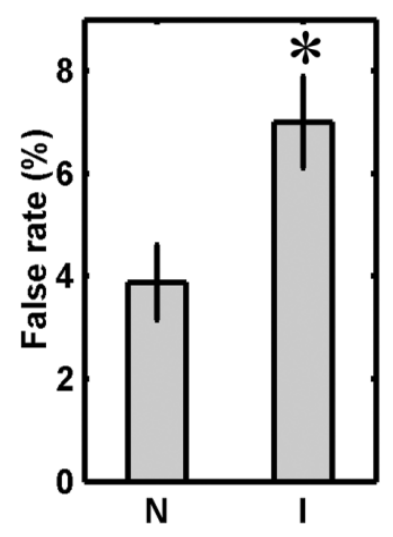

(b)
Fig. 2. Grand average response time (a) and false rate (b) for each stimulus condition. Data are shown in mean \pm standard error (SE). N, neutral stimulus; I, Incongruent stimulus. Black asterisk indicates significant difference. 


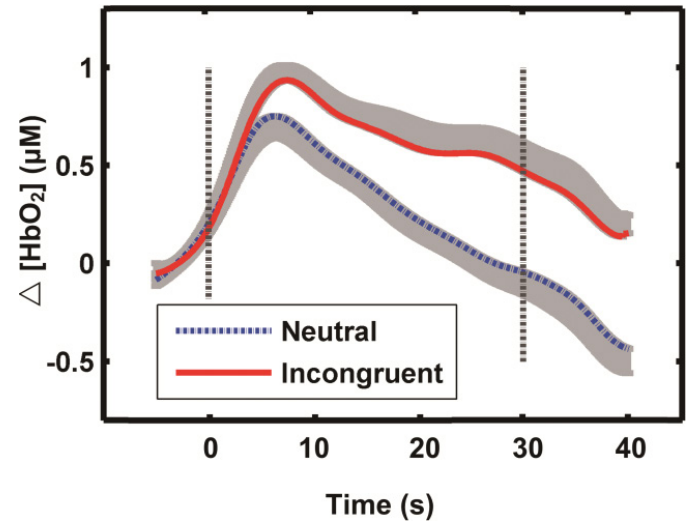

Fig. 3. Grand average time courses of $\mathrm{HbO}_{2}$ signals to incongruent (red) and neutral (blue) tasks at typical Ch. 13. The dotted lines at 0 and $30 \mathrm{~s}$ indicate the start and end of task block. The shadow area indicates SE.

band between 0.07 and $0.50 \mathrm{~Hz}$. Figure 5 displays the coherence changes averaged over all subjects at all homologous channel pairs. The result showed that bilateral functional connectivity was stronger for tasks than rest period, and also stronger for incongruent than neutral tasks. There was significant Stroop effect at Ch. 5-Ch. 13 pair, and marginal Stroop effect at Ch. 8-Ch. 16 pair.

\section{Discussion}

In this study, we measured NIRS signals and recorded the behavioral data at the same time during the Chinese color-word matching Stroop task. The behavioral performance, brain activation and bilateral functional connectivity results showed significant Stroop effects, validating the feasibility of NIRS-based functional connectivity in high-level and complex cognitive tasks.
As to behavioral data, both response time and false rate showed significant Stroop effects, indicating that the design of the experiment was correct.

Considering the limited depth penetration of NIRS, ${ }^{6}$ we chose a color-word matching Stroop task, which activates the bilateral PFC primarily. ${ }^{23,24,33}$ The amplitude of $\mathrm{HbO}_{2}$ signal was higher for incongruent task than neutral task, and showed significant Stroop effects in both left and right PFC. These results of brain activation were consistent with previous studies which reported the involvement of bilateral PFC during the Stroop task. ${ }^{33-35}$ Brain activation results indicated that the recording of NIRS data was correct.

Bilateral PFC functional connectivity was significantly stronger for tasks compared to rest period and for incongruent task compared to neutral task, indicating increased interhemispheric functional integration along with increased workload of brain. This result is consistent with previous studies, which pointed out that interhemispheric functional integration was involved during attentional processing, ${ }^{36}$ and interhemispheric functional integration led to superior performance. ${ }^{37}$ These functional connectivity results proves the feasibility of NIRS-based functional connectivity in the Stroop task.

This study only evaluated functional connectivities of homologous channel pairs. Future studies with bilateral functional connectivity evaluated on the basis of all channel pairs between the left and the right hemispheres are needed to better understand interhemispheric functional integration during cognitive tasks. Also, we just employed WTC method to evaluate functional connectivity, it
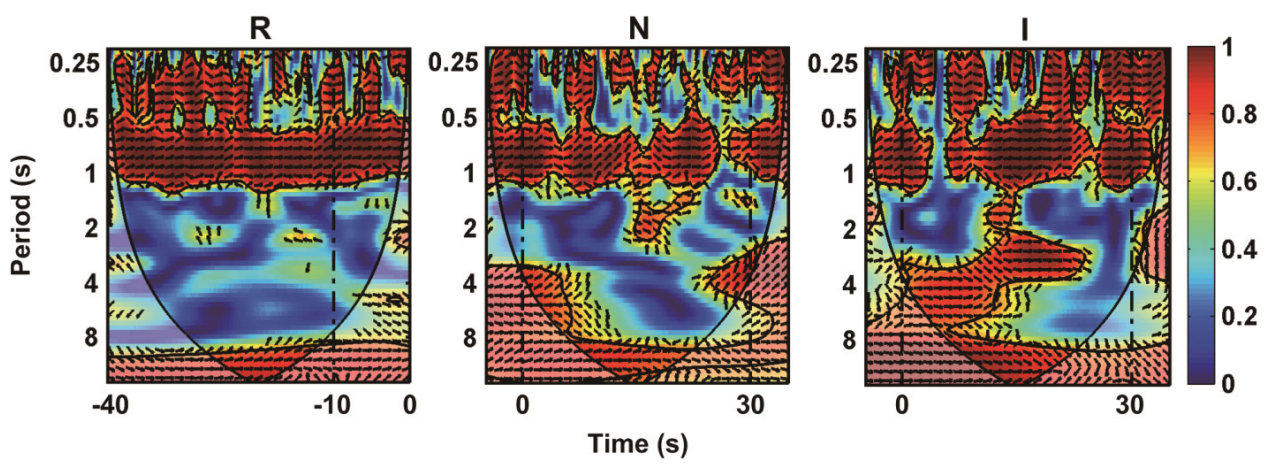

Fig. 4. WTC between the $\mathrm{HbO}_{2}$ signal from channel 5 and channel 13 of a participant. R, rest period; N, neutral task; I, Incongruent task. Black dotted lines at $0 \mathrm{~s}$ indicate start of tasks. We choose time band between 0 and $30 \mathrm{~s}$ to calculate the average coherence value for task blocks, and -40 to $-10 \mathrm{~s}$ for rest period. 
Ch. 1 - Ch. 9

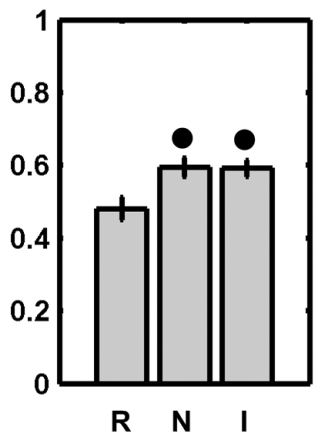

Ch. 5 - Ch. 13

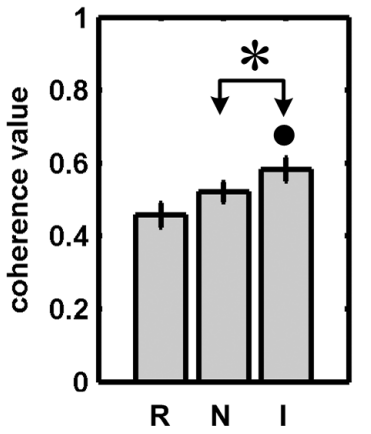

Ch. 2 - Ch. 10

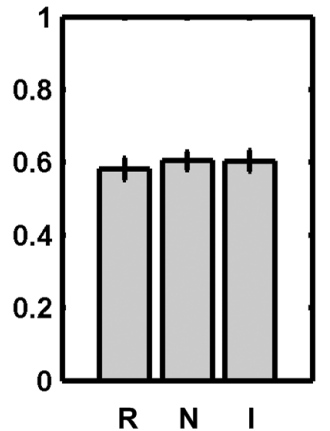

Ch. 6 - Ch. 14

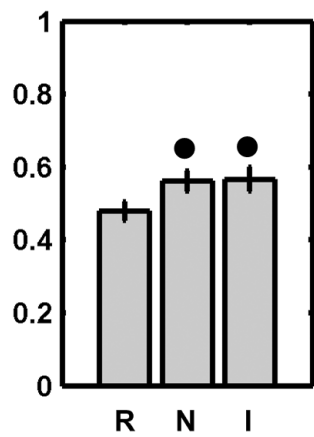

Ch. 3 - Ch. 11

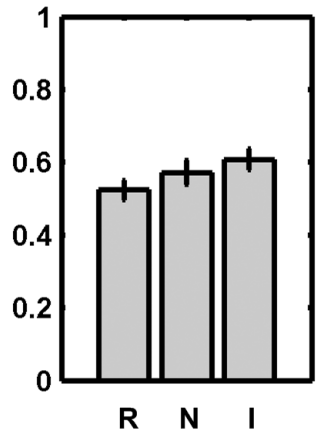

Ch. 7 - Ch. 15

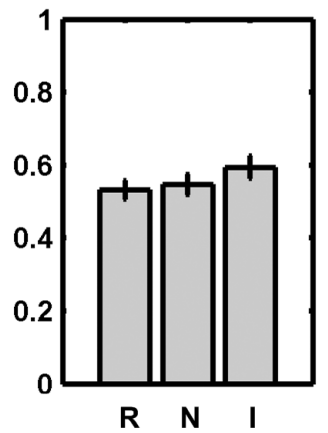

Ch. 4 - Ch. 12

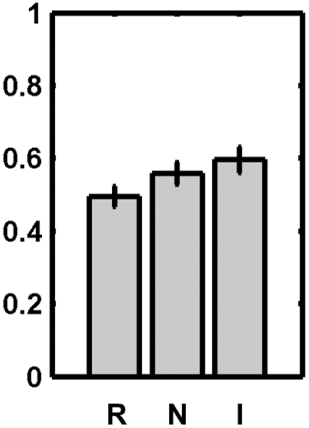

Ch. 8 - Ch. 16

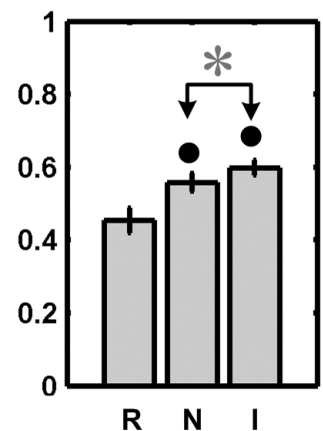

Fig. 5. Grand average coherence value of homologous channel pairs. Data are shown in mean \pm SE. R, rest period; N, neutral task; I, Incongruent task. Significant difference between task and rest period is indicated by black dot $(p<0.05)$. Significant difference between incongruent task and neutral task is indicated by black asterisk $(p=0.004)$ and marginally significant difference is indicated by gray asterisk $(p=0.076)$.

is valuable to investigate the influence of the evaluated method for the detection of NIRS-based functional connectivity in the future.

\section{Conclusion}

Brain functional connectivity can be used to study the organization of brain networks, ${ }^{28}$ characterizing the ways in which multiple brain regions are functionally integrated. ${ }^{5}$ Functional connectivity provides a complimentary measure of brain activity to better understand neural mechanism in cognitive tasks, and it could be even more sensitive than brain activation in some cases. ${ }^{14}$ This study demonstrates that, NIRS becomes a valuable tool to investigate the brain functional connectivity in high-level and complex cognitive tasks.

\section{Acknowledgments}

We would like to thank Qianzhu Luo for proofreading the manuscript. We thank all subjects for their participation. This research was supported by the Science Fund for Creative Research Group of China (Grant No. 61121004), National Natural Science Foundation of China (Grant No. 61078072) and 863 Program (Grant No. 2012AA02A602).

\section{References}

1. Q. Luo, S. Nioka, B. Chance, "Functional nearinfrared imager," Proc. SPIE 2979, 84-93 (1997).

2. M. Ferrari, V. Quaresima, "A brief review on the history of human functional near-infrared spectroscopy (fNIRS) development and fields of application," Neuroimage 63, 921-935 (2012).

3. A. C. Merzagora, M. T. Schultheis, B. Onaral, M. Izzetoglu, "Functional near-infrared spectroscopybased assessment of attention impairments after traumatic brain injury," J. Innov. Opt. Health Sci. 04, 251-260 (2011).

4. G. Strangman, D. A. Boas, J. P. Sutton, "Noninvasive neuroimaging using near-infrared light," Biol. Psychiat. 52, 679-693 (2002).

5. U. Chaudhary, M. Hall, J. DeCerce, G. Rey, A. Godavarty, "Frontal activation and connectivity using near-infrared spectroscopy: Verbal fluency 
language study," Brain Res. Bull. 84, 197-205 (2011).

6. A. Villringer, B. Chance, "Non-invasive optical spectroscopy and imaging of human brain function," Trends Neurosci. 20, 435-442 (1997).

7. G. M. Lech, "Using functional near-infrared spectroscopy to measure cognitive function: When will it become an accepted clinical tool for cognitive aging and prodromal dementia screening?," J. Innov. Opt. Health Sci. 04, 373-383 (2011).

8. S. C. Bunce, M. Izzetoglu, K. Izzetoglu, B. Onaral, K. Pourrezaei, "Functional near-infrared spectroscopy," IEEE Eng. Med. Biol. Mag. 25, 54-62 (2006).

9. B. Chance, Q. Luo, S. Nioka, D. C. Alsop, J. A. Detre, "Optical investigations of physiology: A study of intrinsic and extrinsic biomedical contrast," Philos. Trans. R. Soc. Lond. B 352, 707-716 (1997).

10. K. Izzetoglu, H. Ayaz, A. Merzagora, M. Izzetoglu, P. A. Shewokis, S. C. Bunce, K. Pourrezaei, A. Rosen, B. Onaral, "The evolution of field deployable fNIR spectroscopy from bench to clinical settings," J. Innov. Opt. Health Sci. 04, 239-250 (2011).

11. J. Zhai, T. Li, Z. Zhang, H. Gong, "Hemodynamic and electrophysiological signals of conflict processing in the Chinese-character Stroop task: A simultaneous near-infrared spectroscopy and eventrelated potential study," J. Biomed. Opt. 14, 054022 (2009).

12. M. Smith, "Shedding light on the adult brain: A review of the clinical applications of near-infrared spectroscopy," Philos. Trans. R. Soc. A 369, 44524469 (2011).

13. C. E. Elwell, C. E. Cooper, "Making light work: Illuminating the future of biomedical optics," Philos. Trans. R. Soc. A 369, 4358-4379 (2011).

14. A. V. Medvedev, J. M. Kainerstorfer, S. V. Borisov, J. VanMeter, "Functional connectivity in the prefrontal cortex measured by near-infrared spectroscopy during ultrarapid object recognition," $J$. Biomed. Opt. 16, 016008 (2011).

15. B. Li, F. Zhou, Q. Luo, P. Li, "Altered resting-state functional connectivity after cortical spreading depression in mice," Neuroimage 63, 1171-1177 (2012).

16. H. Niu, S. Khadka, F. Tian, Z. J. Lin, C. Lu, C. Zhu, H. Liu, "Resting-state functional connectivity assessed with two diffuse optical tomographic systems," J. Biomed. Opt. 16, 046006 (2011).

17. R. C. Mesquita, M. A. Franceschini, D. A. Boas, "Resting state functional connectivity of the whole head with near-infrared spectroscopy," Biomed. Opt. Express 1, 324-336 (2010).

18. A. Raz, J. Buhle, "Typologies of attentional networks," Nat. Rev. Neurosci. 7, 367-379 (2006).
19. B. J. Harrison, M. Yucel, M. Shaw, W. J. Brewer, P. J. Nathan, S. C. Strother, J. S. Olver, G. F. Egan, D. Velakoulis, P. D. McGorry, C. Pantelis, "Dysfunction of dorsolateral prefrontal cortex in antipsychotic-naive schizophreniform psychosis," Psychiatry. Res.-Neuroim. 148, 23-31 (2006).

20. A. J. Holmes, D. A. Pizzagalli, "Response conflict and frontocingulate dysfunction in unmedicated participants with major depression," Neuropsychologia 46, 2904-2913 (2008).

21. D. T. Stuss, R. T. Knight, Principles of Frontal Lobe Function, Oxford University Press, New York (2002).

22. F. A. Mansouri, K. Tanaka, M. J. Buckley, "Conflict-induced behavioural adjustment: A clue to the executive functions of the prefrontal cortex," Nat. Rev. Neurosci. 10, 141-152 (2009).

23. S. Zysset, K. Muller, G. Lohmann, D. Y. von Cramon, "Color-word matching stroop task: Separating interference and response conflict," Neuroimage 13, 29-36 (2001).

24. D. G. Norris, S. Zysset, T. Mildner, C. J. Wiggins, "An investigation of the value of spin-echo-based fMRI using a Stroop color-word matching task and EPI at 3 T," Neuroimage 15, 719-726 (2002).

25. B. A. Poser, D. G. Norris, "Application of wholebrain CBV-weighted fMRI to a cognitive stimulation paradigm: Robust activation detection in a stroop task experiment using 3D GRASE VASO," Human Brain Mapp. 32, 974-981 (2011).

26. Z. Zhang, B. Sun, H. Gong, L. Zhang, J. Sun, B. Wang, Q. Luo, "A fast neuronal signal-sensitive continuous-wave near-infrared imaging system," Rev. Sci. Instrum. 83, 094301 (2012).

27. G. Strangman, J. P. Culver, J. H. Thompson, D. A. Boas, "A quantitative comparison of simultaneous BOLD fMRI and NIRS recordings during functional brain activation," Neuroimage 17, 719731 (2002).

28. F. Homae, H. Watanabe, T. Otobe, T. Nakano, T. Go, Y. Konishi, G. Taga, "Development of global cortical networks in early infancy," J. Neurosci. 30, 4877-4882 (2010).

29. A. Duncan, J. H. Meek, M. Clemence, C. E. Elwell, P. Fallon, L. Tyszczuk, M. Cope, D. T. Delpy, "Measurement of cranial optical path length as a function of age using phase resolved near infrared spectroscopy," Pediatr. Res. 39, 889-894 (1996).

30. C. Torrence, G. P. Compo, "A practical guide to wavelet analysis," Bull. Am. Meteorol. Soc. 79, 6178 (1998).

31. X. Cui, D. M. Bryant, A. L. Reiss, "NIRS-based hyperscanning reveals increased interpersonal coherence in superior frontal cortex during cooperation," Neuroimage 59, 2430-2437 (2012). 
32. A. M. Grinsted, J. C. Jevrejeva, "Application of the cross wavelet transform and wavelet coherence to geophysical time series," Nonlinear Process. Geophys. 11, 561-566 (2004).

33. M. L. Schroeter, S. Zysset, T. Kupka, F. Kruggel, D. Y. von Cramon, "Near-infrared spectroscopy can detect brain activity during a color-word matching Stroop task in an event-related design," Human Brain Mapp. 17, 61-71 (2002).

34. T. Egner, A. Etkin, S. Gale, J. Hirsch, "Dissociable neural systems resolve conflict from emotional versus nonemotional distracters," Cereb. Cortex 18, 1475-1484 (2008).
35. M. P. Milham, M. T. Banich, V. Barad, "Competition for priority in processing increases prefrontal cortex's involvement in top-down control: An event-related fMRI study of the stroop task," Brain Res. Cogn. Brain Res. 17, 212-222 (2003).

36. M. T. Banich, "The missing link: The role of interhemispheric interaction in attentional processing," Brain Cogn. 36, 128-157 (1998).

37. M. T. Banich, "Integration of information between the cerebral hemispheres," Curr. Dir. Psychol. Sci. 7, 32-37 (1998). 